\title{
Multisatellite observations of the magnetosphere response to changes in the solar wind and interplanetary magnetic field
}

\author{
Galina Korotova $^{1,2}$, David Sibeck ${ }^{3}$, Scott Thaller ${ }^{4,9}$, John Wygant ${ }^{4}$, Harlan Spence ${ }^{5}$, Craig Kletzing ${ }^{6}$, \\ Vassilis Angelopoulos ${ }^{7}$, and Robert Redmon ${ }^{8}$ \\ ${ }^{1}$ IPST, University of Maryland, College Park, MD, USA \\ ${ }^{2}$ IZMIRAN, Russian Academy of Sciences, Moscow, Troitsk, Russia \\ ${ }^{3}$ NASA/GSFC, Code 674, Greenbelt, MD, USA \\ ${ }^{4}$ College of Science and Engineering, University of Minnesota, Minneapolis, MN, USA \\ ${ }^{5}$ EOS, University of New Hampshire, Durham, NH, USA \\ ${ }^{6}$ Department of Physics and Astronomy, Iowa University, Iowa City, IA, USA \\ ${ }^{7}$ Department of Earth, Planetary and Space sciences, UCLA, Los Angeles, CA, USA \\ ${ }^{8}$ Solar and Terrestrial Physics division, NGDC/NOAA, Boulder, CO, USA \\ ${ }^{9}$ LASP, University of Colorado, Boulder, CO, USA
}

Correspondence: Galina Korotova (gkorotov@umd.edu)

Received: 8 March 2018 - Discussion started: 19 March 2018

Revised: 30 August 2018 - Accepted: 12 September 2018 - Published: 5 October 2018

\begin{abstract}
We employ multipoint observations of the Van Allen Probes, THEMIS, GOES and Cluster to present case and statistical studies of the electromagnetic field, plasma and particle response to interplanetary (IP) shocks observed by the Wind satellite. On 27 February 2014 the initial encounter of an IP shock with the magnetopause occurred on the postnoon magnetosphere, consistent with the observed alignment of the shock with the spiral IMF. The dayside equatorial magnetosphere exhibited a dusk-dawn oscillatory electrical field with a period of $\sim 330$ s and peak-to-peak amplitudes of $\sim 15 \mathrm{mV} \mathrm{m}^{-1}$ for a period of $30 \mathrm{~min}$. The intensity of electrons in the energy range from 31.5 to $342 \mathrm{KeV}$ responded with periods corresponding to the shock-induced ULF (ultralow frequency) electric field waves. We then perform a statistical study of $E_{y}$ variations of the electric field and associated plasma drift flow velocities for 60 magnetospheric events during the passage of interplanetary shocks. The $E_{y}$ perturbations are negative (dusk-to-dawn) in the dayside magnetosphere (followed by positive or oscillatory perturbations) and dominantly positive (dawn-to-dusk direction) in the nightside magnetosphere, particularly near the SunEarth line within an L-shell range from 2.5 to 5. The typical observed amplitudes range from 0.2 to $6 \mathrm{mV} \mathrm{m}^{-1}$ but can reach $12 \mathrm{mV}$ during strong magnetic storms. We show that
\end{abstract}

electric field perturbations increase with solar wind pressure, and the changes are especially marked in the dayside magnetosphere. The direction of the $V_{x}$ component of plasma flow is in agreement with the direction of the $E_{y}$ component and is antisunward at all local times except the nightside magnetosphere, where it is sunward near the Sun-Earth line. The flow velocities $V_{x}$ range from 0.2 to $40 \mathrm{~km} \mathrm{~s}^{-1}$ and are a factor of 5 to 10 times stronger near noon as they correspond to greater variations of the electric field in this region. We demonstrate that the shock-induced electric field signatures can be classified into four different groups according to the initial $E_{y}$ electric field response and these signatures are dependent on local time. Negative and bipolar pulses predominate on the dayside while positive pulses occur on the nightside. The ULF electric field pulsations of Pc and Pi types produced by IP shocks are observed at all local times and in the range of periods from several tens of seconds to several minutes. We believe that most electric field pulsations of the Pc5 type in the dayside magnetosphere at $L<6$ are produced by field line resonances. We show that the direction of the shock normal determines the direction of the propagation of the shock-induced magnetic and plasma disturbances. The observed directions of velocity $V_{y}$ predominately agree 
with those expected for the given spiral or orthospiral shock normal orientation.

\section{Introduction}

Sudden increases in the solar wind dynamic pressure accompanying interplanetary (IP) shocks cause earthward motion of the bow shock and the magnetopause and launch fast and intermediate mode waves into the magnetosphere (Tamao, 1964). The fast mode waves propagate both radially inward and azimuthally around the Earth (Araki et al., 1997), whereas the intermediate mode waves propagate along magnetic field lines to produce transient perturbations in the high-latitude dayside ionosphere (Southwood and Kivelson, 1990; Glassmeier and Heppner, 1992). Using multipoint observations, Wilken et al. (1982) estimated the propagation speeds to be about $600 \mathrm{~km} \mathrm{~s}^{-1}$ in the radial direction from geostationary orbit to the ground and about $910 \mathrm{~km} \mathrm{~s}^{-1}$ in the azimuthal direction in the equatorial plane. Nopper et al. (1982) estimated an impulse disturbance speed of about $1500 \mathrm{~km} \mathrm{~s}^{-1}$ at geostationary orbit. Schmidt and Pedersen (1988) derived a propagation velocity for the radially inward traveling compressive wave of $950 \mathrm{~km} \mathrm{~s}^{-1}$ and for the azimuthal wave in the outer magnetosphere of $1100 \mathrm{~km} \mathrm{~s}^{-1}$. Samsonov et al. (2007) used a magnetohydrodynamic code to simulate the interaction of a moderately strong interplanetary shock propagating along the Sun-Earth line and obtained the average speed of the primary and reflected fast shocks in the magnetosphere of about $700 \mathrm{~km} \mathrm{~s}^{-1}$, in agreement with their assumptions concerning the mean Alfvén velocity in the outer dayside magnetosphere $\left(1000 \mathrm{~km} \mathrm{~s}^{-1}\right)$ and in the plasmasphere $\left(500 \mathrm{~km} \mathrm{~s}^{-1}\right)$.

The IP shock orientation plays an important role in determining the associated geophysical effects. For example, Oliveira and Raeder (2015) showed that system evolution times are much longer for shocks with normals oblique to the Sun-Earth line. The pressure pulse model of Sibeck (1990) predicts dawnward-moving transient events near local noon when shock normals point perpendicular to the nominal spiral interplanetary magnetic field (IMF) direction, but duskward-moving events occur near local noon for events when shock normals point perpendicular to the orthospiral IMF orientation. The direction of the plasma flow within the magnetosphere is expected to be consistent with the orientation of the shock, that is to say dawnward flow for spiral IMF shocks and duskward flow for orthospiral IMF shocks. Here orthospiral refers to IMF longitudes $\left(0^{\circ}<\Lambda<90^{\circ}\right.$ and $\left.180^{\circ}<\Lambda<270^{\circ}\right)$, spiral refers to IMF longitudes $\left(90^{\circ}<\Lambda\right.$ $<180^{\circ}$ and $270^{\circ}<\Lambda<360^{\circ}$, where longitude $\Lambda=0^{\circ}$ points sunward), and $\Lambda=90^{\circ}$ duskward.

The magnetic and electric fields are key parameters for understanding of the response of the Earth's space environment to IP shocks. The propagation and evolution of electric fields in the magnetosphere-ionosphere system in response to IP shocks have been studied for several decades but signatures of the shock-related electric field perturbations are still not fully understood. Knott et al. (1985) reported that the electric field observed by the GEOS-2 satellite showed a transient signature of about $7 \mathrm{mV} \mathrm{m}^{-1}$ in the dayside magnetosphere associated with the onset of a sudden commencement (SC). These signatures were followed by Pc4-5 oscillations. Schmidt and Pedersen (1988) performed a statistical investigation of the GEOS2 electric field signatures associated with SC that showed a clear tailward flow pattern near local noon. Close to the flanks or in the nightside of the magnetosphere the corresponding flows also exhibited a radially inward component. Shinbori et al. (2004) investigated the detailed signatures of the Akebono electric and magnetic fields associated with SCs inside the plasmasphere $(L<5)$. The initial excursion of the electric field associated with SCs was almost directed westward at all local times. The amplitude did not show a clear dependence on magnetic local time, and the intensity of the $E_{y}$ field gradually increased by $0.5-2.0 \mathrm{mV} \mathrm{m}^{-1}$ about $1-2 \mathrm{~min}$ after the onset of the initial electric field impulse. The propagation velocity of SC disturbances derived from the amplitude ratio of the electric field to magnetic field was about $360 \mathrm{~km} \mathrm{~s}^{-1}$ in the equatorial plasmasphere. Kim et al. (2009) used an MHD simulation to examine the electric field and suggested that the SC-associated electric field seen by Shinbory et al. (2004) was the convection electric field. Takahashi et al. (2017) investigated the spatial and temporal evolution of large-scale electric fields in the magnetosphere and ionosphere associated with SCs using multipoint equatorial magnetospheric and ionospheric satellites together with ground radars and showed that the propagation characteristics of electric fields in the equatorial plane depend on magnetic local time. They showed that the initial variation of the electric field (negative $E_{y}$ ) lasted about $1 \mathrm{~min}$ and was directed westward throughout the inner magnetosphere. Positive $E_{y}$ became dominant 2 min after SCs propagated to premidnight or postmidnight regions with near-constant amplitude.

Observations and MHD simulations (e.g., Li et al., 1993; Zong et al., 2009; Halford et al., 2014; Schiller et al., 2016) show that the electric fields generated by sudden compressions can resonantly interact with trapped charged particle populations within the Earth magnetosphere, energizing and injecting them deep into the magnetosphere. During the wellknown shock event in March 1991, the CRESS satellite observed injected electrons energized to extremely high energies, up to $5 \mathrm{MeV}$ (Blake et al., 1992). Wygant et al. (1994) showed that the shock-related electric and magnetic field perturbations observed by the CRRES satellite in the nightside inner magnetosphere exhibited a bipolar waveform with amplitudes of about $80 \mathrm{mV} \mathrm{m}^{-1}$ and $140 \mathrm{nT}$, respectively, and energized the energetic electrons to energies up to $15 \mathrm{MeV}$. Foster et al. (2015) found that a shock with an azimuthal electric field impulse of $10 \mathrm{mV} \mathrm{m}^{-1}$ observed by the Van Allen 
Probes was responsible for accelerating $1.5-4.5 \mathrm{MeV}$ electrons by $400 \mathrm{KeV}$ in the radial region of $L=3.5-4$.

This paper focuses on two major issues. We will inspect multispacecraft electric and magnetic field and particles and plasma observations to study their response to an IP shock on 27 February 2014. We will time the occurrence of magnetic field disturbances associated with the shock in space and the magnetosphere and will show that it propagated dawnward, consistent with expectations based on the shock orientation. Then we will perform a statistical study of the Van Allen Probe electric field disturbances in the magnetosphere and associated plasma drift $V_{x}$ and $V_{y}$ velocities in response to IP shocks. We will show that there are four categories of electric field perturbations that occur in response to shockinduced compressions and that these signatures have a clear dependence on magnetic local time. We will show that the direction of the shock normal has an important effect on the propagation of the shock-induced magnetic and plasma disturbances and that our statistical results are consistent with MHD simulation prediction.

\section{Data sets}

The extensive Van Allen Probes, THEMIS, Cluster and GOES multi-instrument data sets provide numerous opportunities to observe the magnetospheric response to the changes in the solar wind and interplanetary magnetic field monitored by Wind. The five THEMIS spacecraft were launched in 2007 and carry identical instruments and operated in highly elliptical near-equatorial orbits that precess about the Earth with apogees of 12, 20 and $30 \mathrm{Re}$ and orbital periods of 1, 2 and 4 days. With the outermost two spacecraft of ARTEMIS now at the Moon, three THEMIS spacecraft remain on the innermost orbits. We use magnetic field data with $3 \mathrm{~s}$ time resolution from the THEMIS FGM triaxial fluxgate magnetometers (Auster et al., 2008). The ESA electrostatic analyzer on the THEMIS spacecraft measures the distribution functions of 0.005 to $25 \mathrm{keV}$ ions and 0.005 to $30 \mathrm{keV}$ electrons over $4 \pi \mathrm{sr}$ and provides accurate $3 \mathrm{~s}$ time resolution plasma moments, pitch angle and gyrophase particle distributions (McFadden et al., 2008).

The two Van Allen Probes were launched in August 2012 into nearly identical equatorial and low inclination $\left(\sim 10^{\circ}\right)$ orbits with perigee altitudes of 605 and $625 \mathrm{~km}$ and apogee altitudes of 30410 and $30540 \mathrm{~km}$ (Mauk et al., 2012). Both satellites carry identical sets of instruments to measure charged particle populations, fields and waves in the inner magnetosphere. In this paper, we employ observations from the Energetic Particle, Composition, and Thermal Plasma Suite (ECT: MagEIS, 20-4000 keV for electrons) (Spence et al., 2013; Blake et al., 2013), Electric and Magnetic Field Instrument Suite and Integrated Science (EMFISIS) (Kletzing et al., 2013), and the Electric Field and Waves Suite (EFW) (Wygant et al., 2013). In particular, we inspect elec-

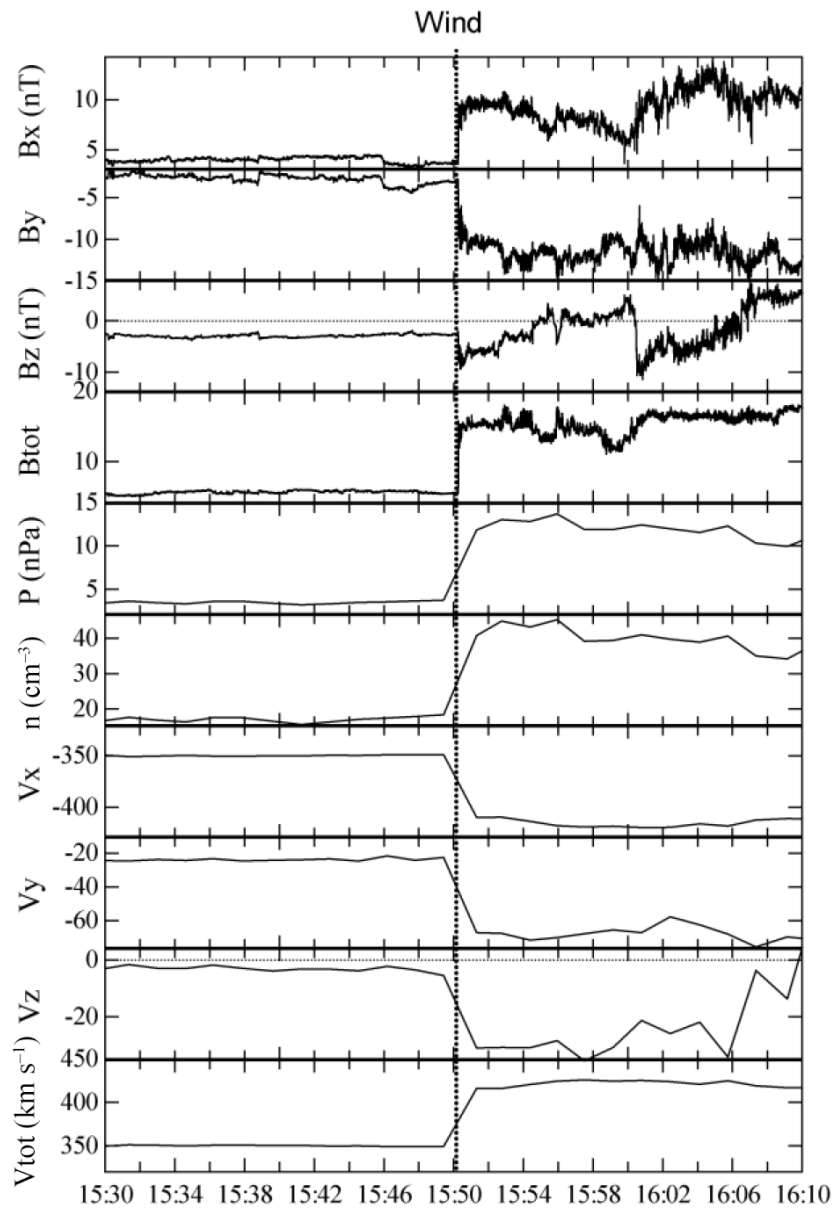

Figure 1. Wind observations of magnetic field and plasma in GSM coordinates from 15:30 to 16:10 UT on 27 February 2014. Dashed line shows the time of arrival of an interplanetary shock.

tric and magnetic field observations with 11 and $4 \mathrm{~s}$ time resolution, respectively, and differential particle flux measurements with $\sim 11 \mathrm{~s}$ (spin period) time resolution. The electric field data were obtained from sites http://www.space. umn.edu/rbspefw-data (Wygant and Breneman, 2017) and CDAWEB (NASA, 2018), where they are presented in an MGSE (modified GSE) coordinate system. They provide two components $Y$ and $Z$ of the electric field. Both components are in the spin plane of the spacecraft and are measured with the $50 \mathrm{~m}$ long booms. The spin axis $X$ is oriented within $37^{\circ}$ of the Earth-Sun line. The spin axis component of the electric field can be obtained from the $E \cdot B=0$ assumption. For this to succeed the magnetic field should be at least $15^{\circ}$ out of the spin plane. To calculate Van Allen Probe plasma flow velocities we converted the electric field data from modified MGSE coordinates into GSE coordinates. Additionally we used magnetic field data from GOES 13 and 15 with $0.5 \mathrm{~s}$ time resolution (Singer et al., 1966) and Cluster with $4 \mathrm{~s}$ time resolution (Balogh et al., 1997). We use Wind solar wind 
Table 1. Times of encounter of the IP shock with the spacecraft and their locations in GSM coordinates.

\begin{tabular}{lr|rrr}
\hline Spacecraft & Time & \multicolumn{3}{|c}{ Position } \\
\hline Wind & $15: 50: 12$ & 220.90 & 93.92 & 31.49 \\
Cluster 1 & $16: 48: 46$ & 13.10 & 7.82 & -9.44 \\
Cluster 3 & $16: 48: 57$ & 12.60 & 7.73 & -10.16 \\
THEMIS D & $16: 49: 04$ & 11.03 & 0.48 & 1.10 \\
THEMIS A & $16: 49: 12$ & 9.22 & 4.39 & 0.53 \\
Probe A & $16: 50: 33$ & 4.86 & -1.69 & 0.12 \\
Probe B & $16: 50: 26$ & 5.33 & -1.39 & -0.10 \\
GOES 13 & $16: 50: 07$ & 6.51 & -0.60 & 0.99 \\
GOES 15 & $16: 50: 40$ & 2.71 & -6.02 & 0.45 \\
\hline
\end{tabular}

magnetic field and SWE plasma data with $3 \mathrm{~s}$ (Lepping et al., 1995) and $1 \mathrm{~min}$, respectively (Ogilvie et al., 1995).

\section{Observations}

Figure 1 presents Wind magnetic and plasma data from 15:30 to 16:10 UT on 27 February 2014. The arrival of the shock at Wind at 15:50 UT $(X, Y, Z$ GSM $=(220.9,93.9,30.7 \mathrm{Re}))$ is revealed by an enhancement in the interplanetary magnetic field strength from 6 to $16 \mathrm{nT}$ and total plasma velocity from 350 to $420 \mathrm{~km} \mathrm{~s}^{-1}$. The IMF had positive $B_{x}$ and negative By components during the whole interval that both increased the shock the arrived. The solar wind density increased from 18 to $45 \mathrm{~cm}^{-3}$, and the dynamic pressure increased from 3 to $13 \mathrm{nPa}$. This fast forward (FF) shock was oblique. Its normal was calculated using magnetic field coplanarity and pointed in the GSM $\left[n_{x}, n_{y}, n_{z}\right]=[-0.8,-0.4,-0.3]$ direction, i.e., antisunward, dawnward and southward. Consequently the shock should first strike the northern dusk bow shock and magnetopause. i.e., it has a spiral IMF orientation. We will use the direction of the shock normal to interpret the timing results for the IP shock arrival observed by THEMIS, GOES, Cluster and the Van Allen Probe spacecraft for this event.

Figure 2 shows the GSM locations of the THEMIS, Cluster, Van Allen Probes and GOES spacecraft at $\sim 16: 50$ UT (their coordinates are given in Table 1.) All the spacecraft located in the solar wind observed the enhanced magnetic field strength, densities, velocities and temperatures associated with the IP shock. The shock-induced disturbances were seen just upstream from the bow shock by Cluster 1 and 3, located at high southern postnoon latitudes at 16:48:46 and 16:48:57 UT, respectively.

Figure $3 \mathrm{a}, \mathrm{b}$ show the THEMIS D and A observations of the magnetic field, plasma and energy spectra of ion fluxes from 16:40 to 17:20 UT. The spacecraft were initially located in the magnetosheath. At 16:49:04 UT the IP shock hit THEMIS D as indicated by enhanced densities, magnetic field strength and velocities. Particles from low to high en-
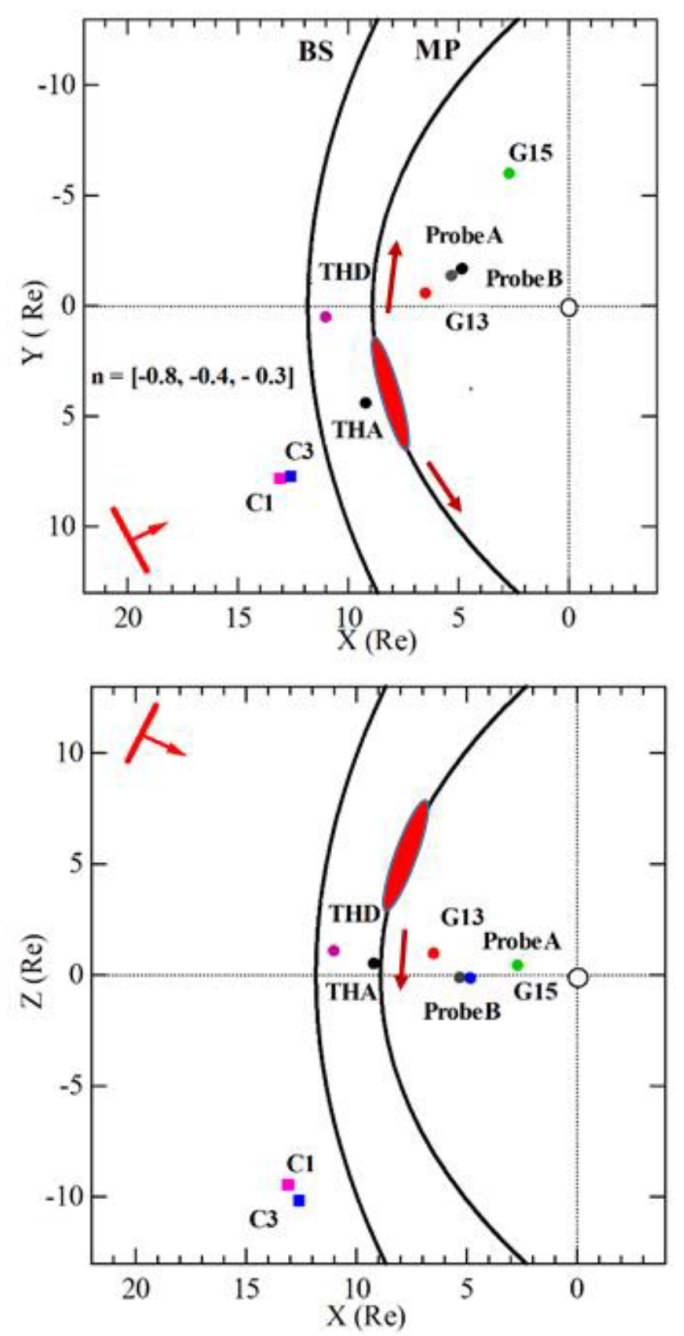

Figure 2. GSM locations of Cluster 1 and 3, THEMIS A and D, Van Allen Probes A and B, and GOES 13 and 15 in the $X-Y$ and $Z-Y$ GSM planes at $\sim 16: 50$ UT on 27 February 2014. The meaning of the solid oval and thick arrows will be discussed in the text later.

ergies showed the increase in energy and enhanced fluxes. The shock-produced compression caused the bow shock to move inward at 16:49:36 UT, past the spacecraft, as indicated by the decrease in the magnetic field strength and decrease in density and temperature and spectra expected for its entry into the solar wind. THEMIS A observed the IP shock at 16:49:12 UT and about $1 \mathrm{~min}$ and $34 \mathrm{~s}$ later its magnetic field, density and temperature traces indicate that the bow shock moved inward past THEMIS A.

Figure $4 \mathrm{a}, \mathrm{b}$ show GOES 13 and 15 observations of the magnetic field (NOAA, 2018) from 16:40 to 17:20 UT. Following the arrival of the transmitted IP shock at GOES 13 near local noon at 16:50:07 UT, there was a sharp increase in magnetic field variations, with amplitudes of $\sim 70 \mathrm{nT}$ in the $H$ component. The shock-induced compression was so 
(a)

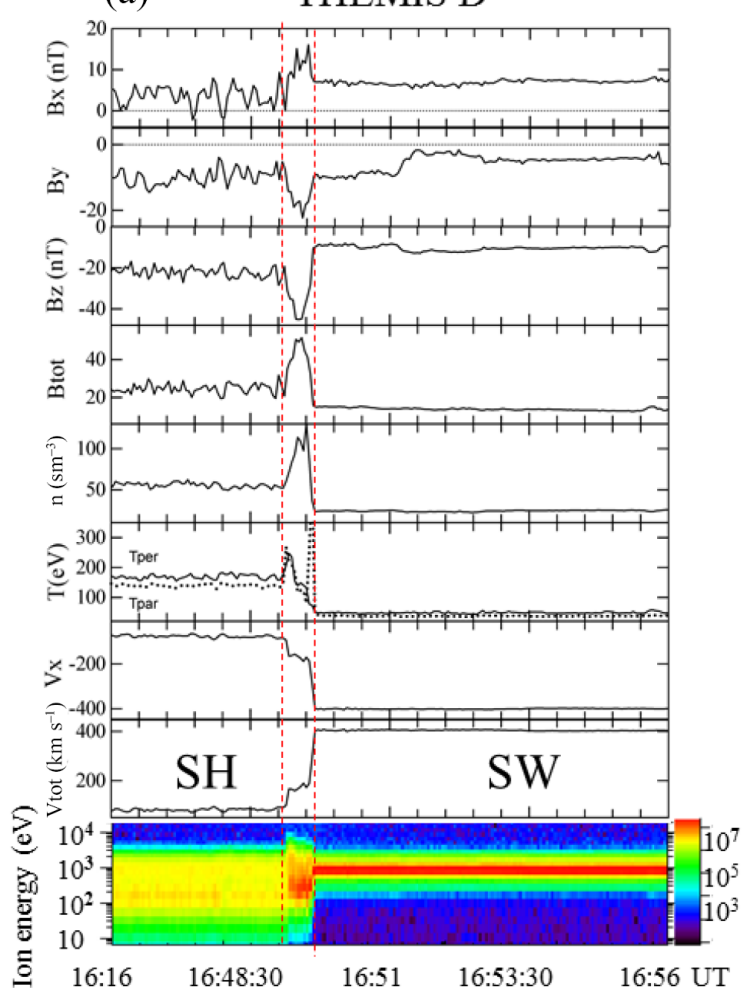

(b)

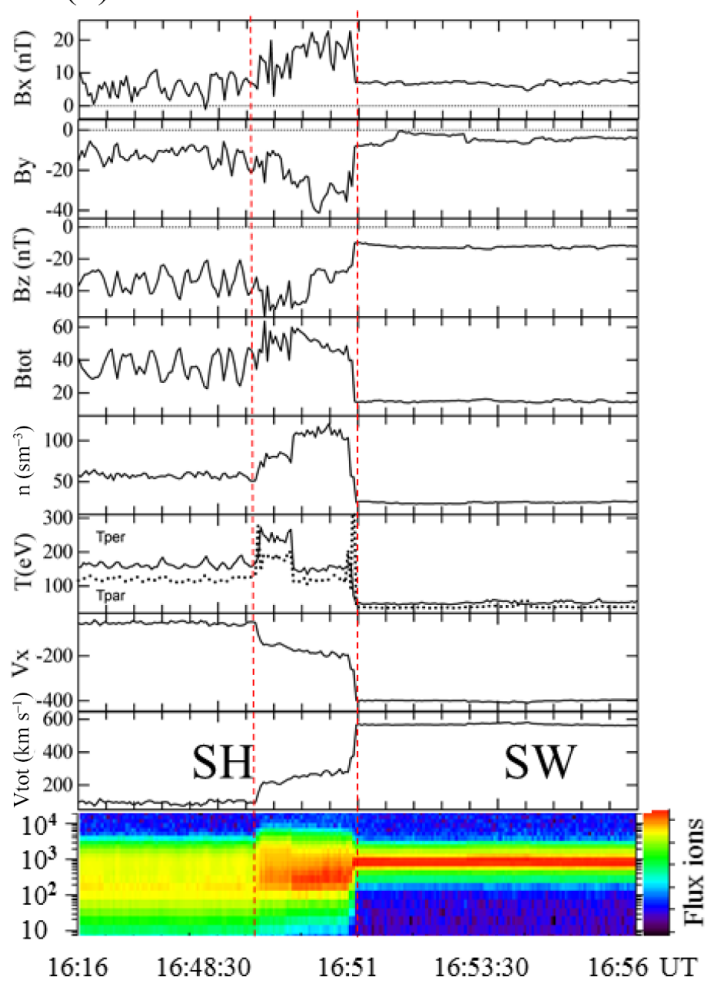

Figure 3. THEMIS A (a) and THEMIS D (b) observations of the magnetic field in GSM coordinate plasma and energy spectra of ion fluxes from 16:16 to 16:56 UT on 27 February 2014. At 16:49:01 UT the IP shock hit THEMIS D as indicated by enhanced densities, magnetic field strength and velocities. Particles from low to high energies showed the increase in energy and enhanced fluxes. The shock-produced compression caused the bow shock to move inward at 16:49:36 UT, past the spacecraft, as indicated by the decrease in the magnetic field strength, density, temperature, and spectra expected for its entry into the solar wind. THEMIS A observed the IP shock at 16:49:12 UT and about 1 min and $34 \mathrm{~s}$ later its magnetic field, density and temperature traces indicate that the bow shock moved inward past THEMIS A.

strong that at 17:02 UT GOES 13 briefly entered the sheath. The shock front was then detected at GOES 15 in the morning (local time) $33 \mathrm{~s}$ later at 16:50:40 UT, where it caused a gradual increase in the magnetic field amplitudes by $\sim 20 \mathrm{nT}$ followed by compressional pulsations that fall in the category of Pc5 pulsations.

The upper and middle panels of Fig. 5a, b present the Van Allen Probes A and B magnetic field and electric observations from 16:40 to 17:20 UT. The arrival of the shock characterized by a strong $(\sim 50 \mathrm{nT})$ increase in the total magnetic field strength and bipolar variations in all three components of the electric field at $\sim 16: 50: 26$ UT at Probe B and $7 \mathrm{~s}$ later at Probe A. The initial electric field perturbations in the $E_{y}$ component observed by Van Allen Probes A and $\mathrm{B}$ were directed dawnward with amplitudes of -9.4 and $-8.2 \mathrm{mV} \mathrm{m}^{-1}$, respectively, but $\sim 4$ min later the sense changed direction towards dusk (with amplitudes of 5.3 and $5.8 \mathrm{mV} \mathrm{m}^{-1}$ ). We interpret these variations as due to a compression of the magnetosphere followed by a reflection (Samsonov et al., 2007). The Ez and Ex components show variations with amplitudes that are a factor of 1.5-2 smaller than those of the $E_{y}$ component. The bipolar electric field waveforms are followed by geomagnetic pulsations with periods of $\sim 330$ s that damp within $\sim 30 \mathrm{~min}$.

Figure $6 \mathrm{a}, \mathrm{b}$ present Van Allen Probes A and B observations of the $E_{y}$ component of the electric field and pitch angle distributions for electron energies of 31.5, 53.8, 108.3, 183.4, 231.8 and $342 \mathrm{KeV}$ measured by the MagEIS instrument. The electrons exhibit enhanced intensities at all energies but the most intense occur at pitch angles near $90^{\circ}$, immediately after the arrival of the IP shock. Kanekal et al. (2016) suggested that the shock-injection mechanism can be effective for energizing particles over a substantial range of pitch angles. The initial flux enhancement is more pronounced by comparison with the following pulses. One of the interesting features in Fig. $6 a, b$ is that the intensity of electrons in the energy range of $31.5-342 \mathrm{KeV}$ exhibits a regular periodicity with periods corresponding to the ULF electric field waves. The oscillations in electron fluxes are in quadrature with the $E_{y}$ component. This component is of special interest because some charged particles that drift azimuthally as a consequence of the gradient and curvature 
(a)

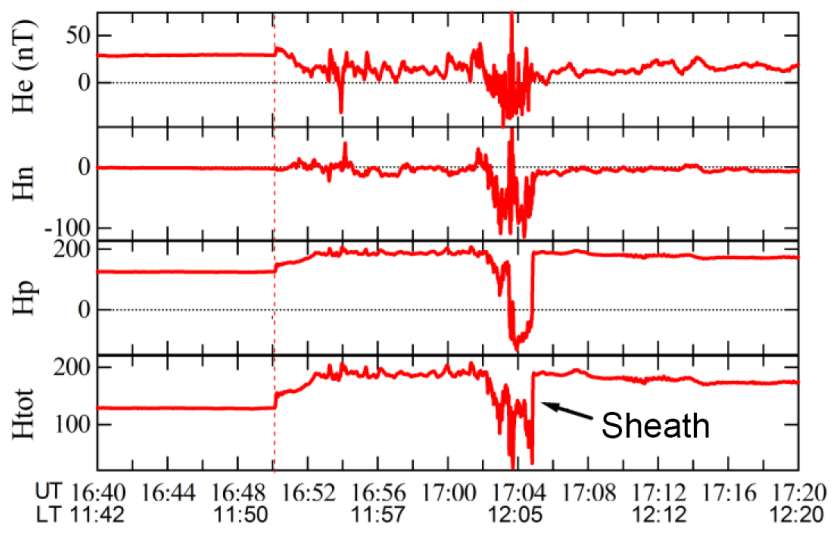

(b)

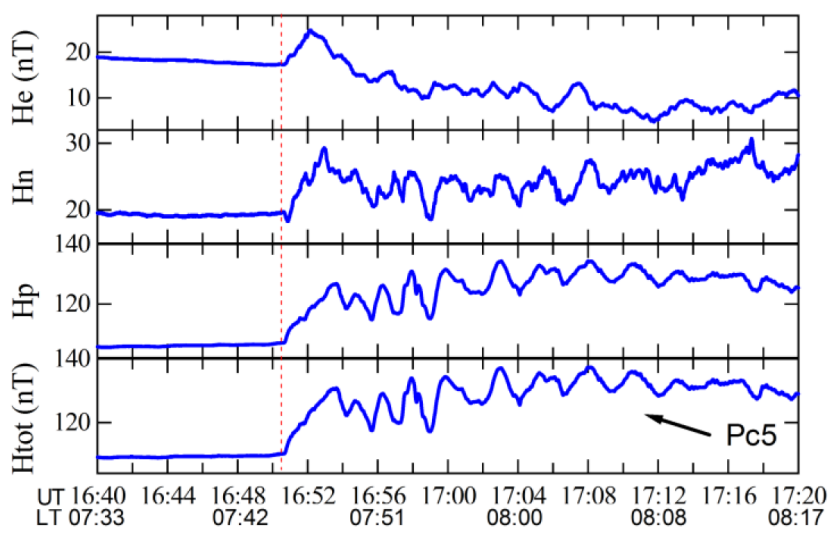

Figure 4. GOES 13 (a) and GOES 15 (b) magnetic field observations in PEN coordinates from 16:40 to 17:20 UT on 27 February 2014. $H_{\mathrm{p}}$ is perpendicular to the satellite's orbital plane, $H_{\mathrm{e}}$ pointing earthward parallel to the satellite-Earth center line, and $H_{n}$ is perpendicular to both $H_{\mathrm{p}}$ and $H_{\mathrm{e}}$ and pointing eastward.

drifts in the Earth magnetic field can traverse this electric field, acquiring a significant amount of energy. Figure 7a$f$ present the response of the energetic electrons to the IP shock in the energy range from 31 to $183 \mathrm{keV}$. Panels $\mathrm{d}$ and b show that after the shock arrival the electron population increased, especially for the lower energies. In the electrical field of $15 \mathrm{mV} \mathrm{m}^{-1}$ electron fluxes increased by factors of 21 and 14 at Van Allen Probes B and A, respectively, in less than a drift period (panels e and $\mathrm{f}$ ). The energetic electron fluxes do not display obvious phase differences across the energies. We interpret these observations as evidence of the prompt energization of electrons due to shock-induced ULF electric fields with an additional contribution for the initial acceleration from the compressional effect of the shock. It should be noted that electrons can be accelerated most significantly via drift resonance (Southwood and Kivelson, 1981) when resonant particles drift with the same velocity as the wave front. Claudepierre et al. (2013) showed Van Allen Probe ob- servations of the energy dependence of the amplitude and phase of the electron flux modulations, which were consequences of drift resonance between $\sim 60 \mathrm{keV}$ electrons and fundamental poloidal Pc5 waves. Hao et al. (2014) presented Van Allen Probe observations of electron injections caused by the IP shock and showed that the injected electrons with energies between $150 \mathrm{KeV}$ and $230 \mathrm{KeV}$ were in drift resonance with the excited poloidal ULF waves. Considering the process for energizing drift-resonant electrons, the value for the $E \times B$ drift velocities of the particles in the wave fields provides important information. We calculated the $V_{x}$ and $V_{y}$ drift velocities at Van Allen Probes A and B for the interval from 16:40 to 17:20 UT and present them in the two bottom panels of Fig. 5a, b. The $V_{x}$ and $V_{y}$ components associated with the minimum peak of the $E_{y}$ electric field are about -40 and $-15 \mathrm{~km} \mathrm{~s}^{-1}$ for Van Allen Probe B and -35 and $-6 \mathrm{~km} \mathrm{~s}^{-1}$ for Van Allen Probe A, i. e., the initial direction of the plasma flow is tailward and dawnward, consistent with expectation for the spiral orientation of the IP shock.

Interaction with the initial fast mode pulse and subsequent ULF electrical field oscillations can have an important effect on particle acceleration. In considering the energization of electrons on 27 February 2014, an encounter with the observed electric field for a period of $240 \mathrm{~s}$ will transport the electrons earthward by $\delta \mathrm{Re}=1.3$ to $1.6 \mathrm{Re}$ from their original position at $L=6.4$ for Van Allen Probe A and at $L=7.1$ for Van Allen Probe B. Conservation of the first adiabatic invariant implies that such particles will be energized by a factor of about 1.9-2.3 in only one cycle of the electric field pulsations. The studies of Wygant et al. (1994), using CRRES data, and Foster et al. (2015), using Van Allen Probe data, and others have demonstrated that the tailward propagation of the strong shock-induced electric field impulse and subsequent ULF processes can result in the extremely fast acceleration of relativistic electron populations inside the plasmasphere.

Knowing the distances between the satellites and the lag times for the propagation of shock-induced disturbances, we calculated the shock propagation velocities. Table 1 summarizes the onset times of the shock-driven encounters of different spacecraft. In the solar wind Cluster 1 observed the shock earlier than Cluster 3, respectively, i.e., the shock moved dawnward. The shock perturbations occurred almost simultaneously in the magnetosheath at Themis $\mathrm{A}$ and $\mathrm{D}(\delta t<10 \mathrm{~s})$ suggesting the front strikes a broad region of the magnetopause at once. The shock-induced impulse propagated antisunward, southward, and both dawnward and, presumably, duskward (thick arrows in Fig. 2) from the point of origin on the magnetopause (depicted as a red oval in Fig. 2), which is consistent with the orientation of the IP shock. In the outer magnetosphere the propagation velocity for the disturbance was about $1348 \mathrm{~km} \mathrm{~s}^{-1}$ between Goes 13 and 15 but only about $390 \mathrm{~km} \mathrm{~s}^{-1}$ between Van Allen Probes B and A. We believe that the shock-induced pulse propagated with the velocity of fast mode waves. The local fast mode speed can be 
(a)

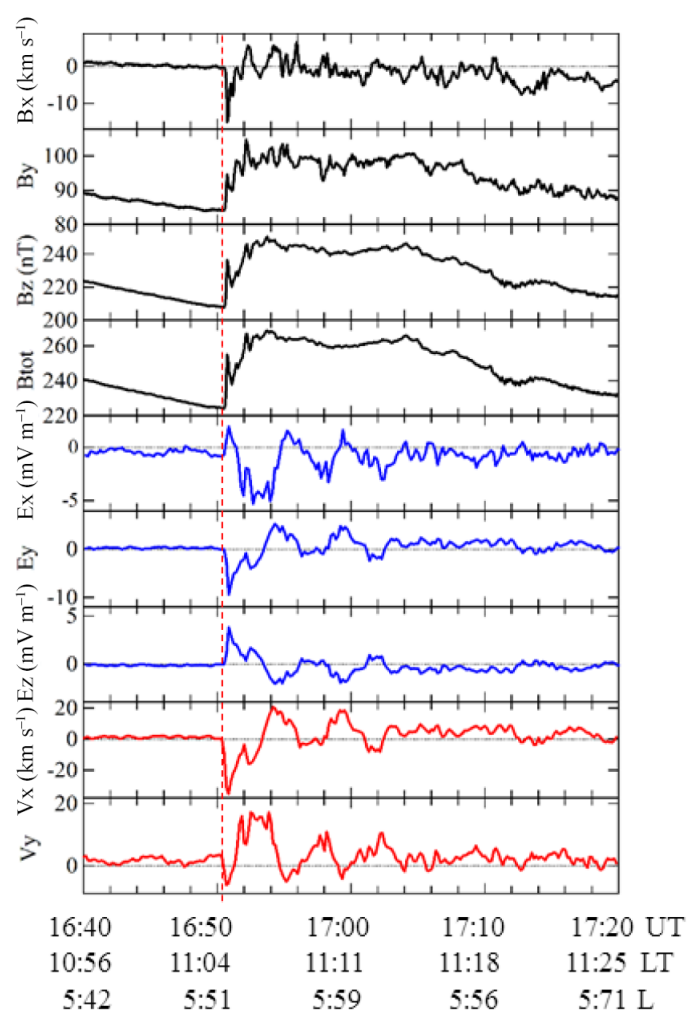

(b)

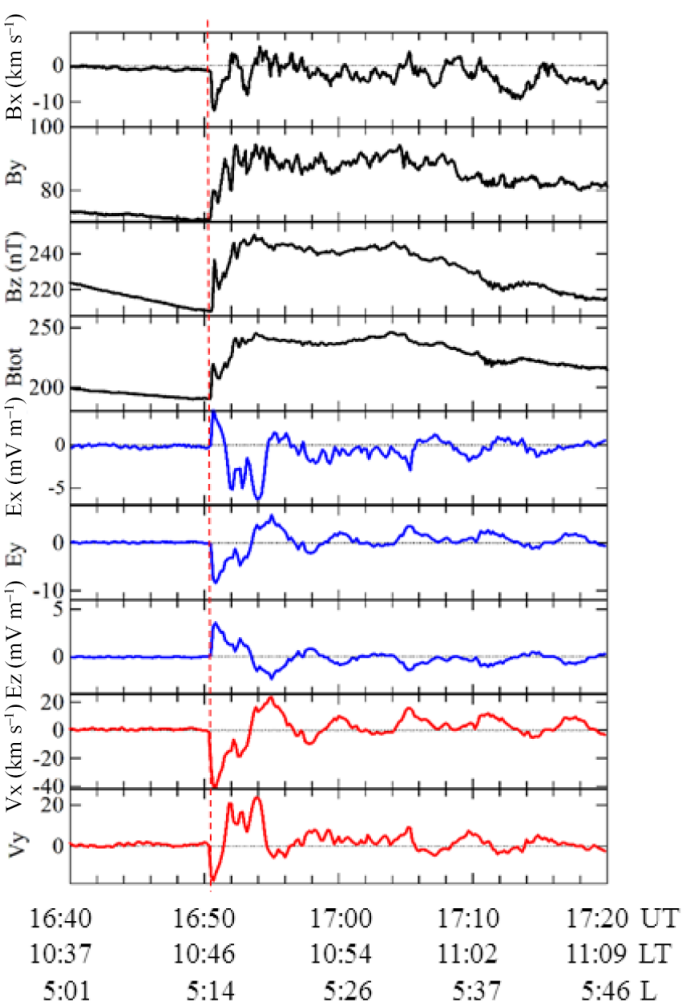

Figure 5. Van Allen Probes A (a) and B (b) magnetic and spin-fit electric field observations and the $V_{x}$ and $V_{y}$ plasma flow velocities in GSE coordinates from 16:40 to 17:20 UT on 27 February 2014.

evaluated from Van Allen Probe measurements of the magnetic field and density. At the time of the shock encounter Van Allen Probes A and B were in the high-density plasmasphere at $L=5.5$ and $L=5.1$, respectively. For a measured local magnetic field of $255 \mathrm{nT}$ for Probe A and $220 \mathrm{nT}$ for Probe $\mathrm{B}$ and density of $\sim 200 \mathrm{~cm}^{-3}$ derived from the potential of both spacecraft, the fast-mode speeds will be $\sim 395$ and $337 \mathrm{~km} \mathrm{~s}^{-1}$, respectively, which are consistent with our estimates of the propagation velocity derived from the time difference of shock arrivals at the spacecraft. The decrease in the fast mode wave speed in the plasmasphere relative to that in the outer magnetosphere agrees well with earlier studies (e.g., Wilken, 1982; Foster et al., 2016).

\section{Statistical study of shock-initiated signatures of the electric field}

The list of IP shocks used in this study was obtained from the Heliospheric Shock Database maintained and generated by the University of Helsinki (2018). They identify shocks by visual inspection and an automated shock-detection algorithm. To be included in the database a shock should satisfy the following upstream to downstream jump conditions: $B_{\text {down }} / B_{\text {up }}>1.2, N_{\text {down }} / N_{\text {up }}>1.2, T_{\text {down }} / T_{\text {up }}>1 / 1.2$, for FF
$V_{\text {down }}-V_{\text {up }}>20 \mathrm{~km} \mathrm{~s}^{-1}$. The normal vector of the shock ( $n$ ) was calculated from the magnetic field data and velocities using the mixed-mode method (Abraham-Shrauner and Yun, 1976). When there is data gap in the velocity components the normal was calculated using magnetic field coplanarity (Colburn and Sonett, 1966).

In view of the importance of the electric field in energizing particles, we performed a statistical study of $E_{y}$ variations of the electric field and associated plasma drift $V_{x}$ and $V_{y}$ velocities during the passage of interplanetary shocks. We identified more than 60 events observed by Van Allen Probes A and B associated with FF IP shocks for the period from 2013 to 2015. The shocks arrived from Wind with lag times in the time range from 26 to $58 \mathrm{~min}$ and produced magnetic field perturbations in the magnetosphere from several to $130 \mathrm{nT}$. Discontinuities in the solar wind plasma such as shocks have often been considered as possible triggers for the release of energy stored within the magnetotail in the form of magnetospheric substorms. Most previous studies of shocks leading to substorms have relied on ground magnetometer observations. Recently it has been shown that the use of global auroral images to identify substorm onsets has some advantages over many other alternative substorm onset signatures, such as low-latitude Pi2 pulsations, auroral kilometric radi- 
(a) Probe $\mathrm{A}$

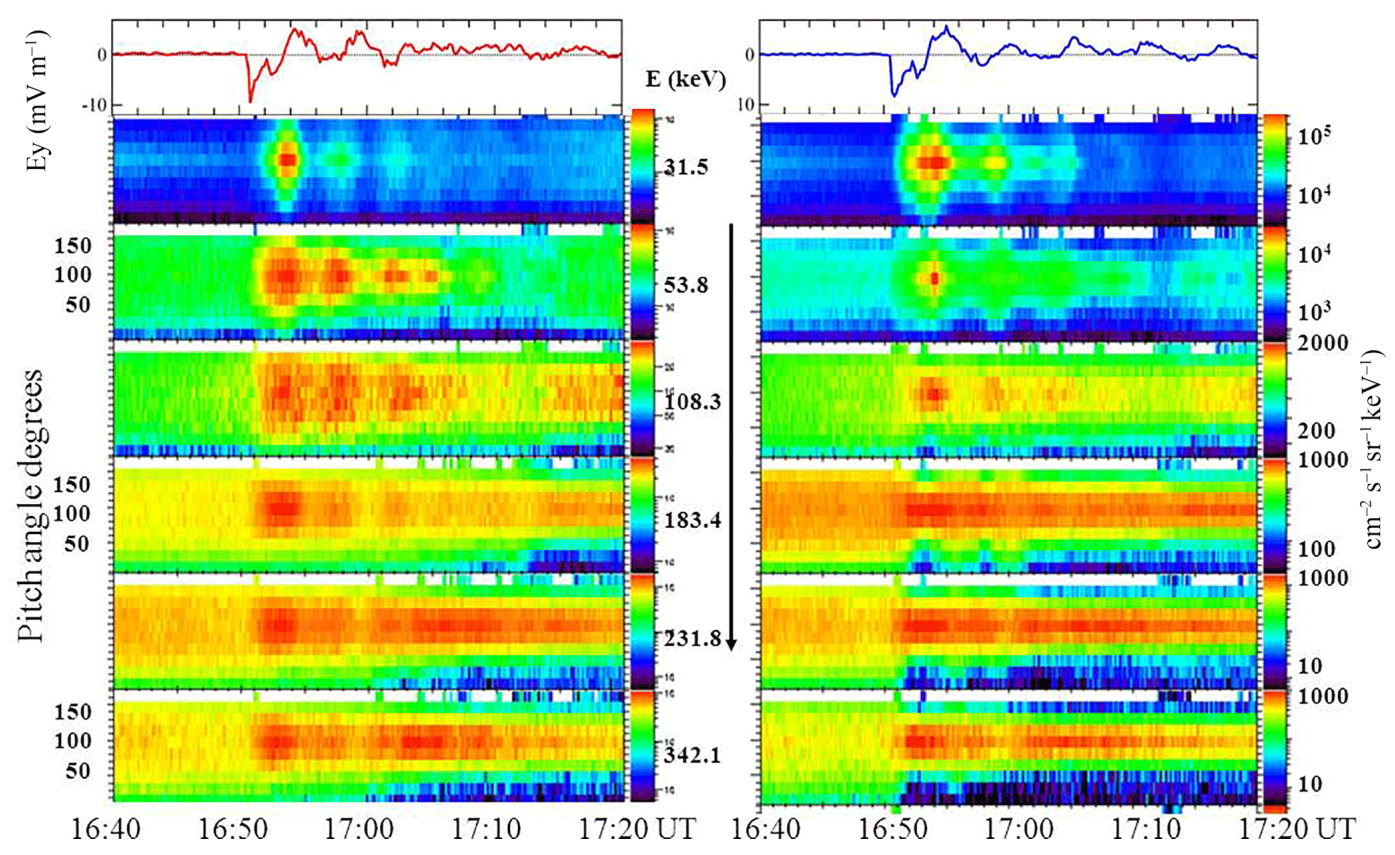

Figure 6. Van Allen Probes A (a) and B (b) $E_{y}$ component of the electric field and pitch angle distributions of electron fluxes in the range of energies from 31.5 to $342 \mathrm{KeV}$, measured by MagEIS instrument from 16:40 to 17:20 UT on 27 February 2014. The log fluxes are color coded according to the color bar shown in the right panel.

ation (AKR) and dispersionless particle injections and magnetic field dipolarization at geosynchronous orbits (e.g., Liou et al., 2000). To identify substorms triggered by shocks in our study we considered negative magnetic bays by examining the westward auroral electrojet $\mathrm{AL}$ index at the times when SSC were determined from low-latitude ground magnetograms. As a quantitative definition for the substorm bay does not exist we used the criteria of Liou et el. (2003) that there should be a sharp decrease in AL of at least $100 \mathrm{nT}$ occurring within a 20 min window starting at the SSC. We found that shocks triggered a substorm in the magnetosphere in 17 of the 30 examined events. Further study of whether these negative magnetic bays are unique identifiers of substorms is beyond the scope of the paper. Other effects in the magnetosphere initiated by IP shocks are perturbations in the electric field (Wygant et al., 1994) and the radiation belt (Blake et al., 2013). Understanding and predicting such responses is important for reducing the risks associated with space exploration. We found that 55 events showed an electron enhancement at energies of $32-54 \mathrm{keV}$, measured by MagEIS at local time, and three of them were accompanied by intensity decreases at higher energies. Five events showed a decrease in the $32-54 \mathrm{keV}$ energy electrons observed in the nightside magnetosphere.

The passage of a shock causes electric field perturbations and their amplitudes to increase in proportion to the intensity of the IP shocks. The E field vectors prior to each com- pression differ greatly from those during the compressional activity. We classified the shock-induced electric field signatures into four different groups according to the examples presented in the upper panels of Fig. 8. Group A presents a negative pulse in the $E_{y}$ component, B group presents a negative-positive pulse, $C$ group presents a positive pulse and D group presents pulsations. Figure 9 presents occurrence patterns for events with the four different signatures of the electric field initiated by IP shocks. It provides evidence that they are dependent on local time. Negative and bipolar pulses predominate on the dayside while positive pulses occur on the nightside. The ULF electric field pulsations of Pc and Pi types produced by IP shocks are observed at all local times and in the range of periods from several tens of seconds to several minutes. We believe that the magnetic field as well the electric field pulsations initiated by IP shocks are generated by a wide variety of mechanisms including plasma instabilities, transient reconnection and pressure pulses and often correspond to field line resonances. Their characteristic features are determined to large extent by local time. In the dayside magnetosphere typical pulsations are of the Pc5 type. Sometimes they last for more than 20 wave cycles without noticeable damping, which could be explained by a continuous input of the solar wind energy into the magnetosphere. In the nightside magnetosphere during substorms, the generation of Pi2 pulsations is more common. They exhibit an irregular form, last 3-5 wave cycles and often exhibit damp- 

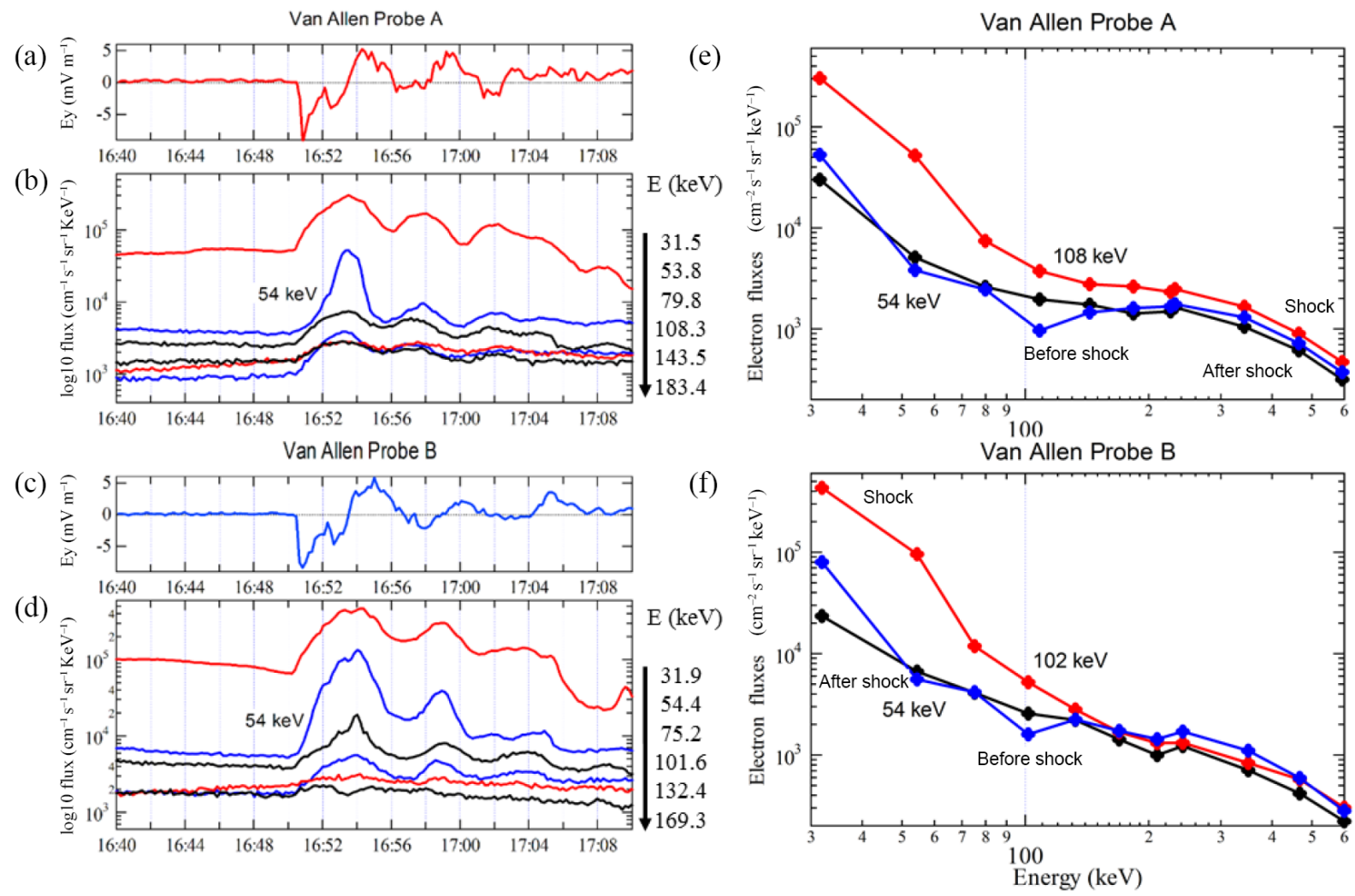

Figure 7. Response of the energetic particles to the transmitted IP shock. Panels (a) and (c) show measurements of the $E_{y}$ component of the electric field. Panels (b) and (d) show electron fluxes for the energies ranging from 31.5 to $180 \mathrm{keV}$ at Van Allen Probes A and B. Panels (e) and (f) show energetic electron spectra observed at Probes A and B before the shock (at 16:48 UT), after the first peak (at 16:53 UT) and 18 min after the shock (at 17:08 UT).

ing. Figure 10 presents periods of the pulsations (measured for the first wave cycle of oscillations) as a function of radius and shows that periods increase with increasing radius. A simple explanation for this behavior of pulsation frequencies with radial distance can be given in terms of standing Alfvén waves along resonant field lines (Sugiura and Wilson, 1964). The length of the field lines, the magnetic field strength and the plasma density distribution determine the Alfvén velocity, and the periods of the pulsations. This plot indicates that most electric field pulsations of the Pc5 type in the dayside magnetosphere at $L<6$ are produced by field line resonances.

Figure 11 presents the amplitudes and direction of the initial $E_{y}$ response to IP shocks in the $X-Y$ GSM plane. The perturbations are negative (dusk-to-dawn) in the dayside magnetosphere (followed by the positive or oscillatory perturbations) and dominantly positive (dawn-to-dusk direction) in the nightside magnetosphere, in particular mostly near the Sun-Earth line within an $L$-shell range from 2.5 to 5 . The typical observed amplitudes range from 0.2 to $6 \mathrm{mV} \mathrm{m}^{-1}$ but can reach $12 \mathrm{mV}$ during strong magnetic storms. In the nightside magnetosphere the response of $E_{y}$ is rather weak and its amplitudes do not exceed $3 \mathrm{mV} \mathrm{m}^{-1}$. To demonstrate the impact of IP shocks Fig. 12 shows amplitudes of the initial electric field variations (blue and red crosses) as a function of dynamic pressure observed at Wind. The electric field perturbations increase with the solar wind pressure and that the changes are especially marked in the dayside magnetosphere (red points) as this region is more fully exposed to compression than the nightside sector that is shielded from the frontside compression.

To determine the $V_{x}$ direction of the plasma after the impact of IP shocks we used the formula $V=E \times B / B^{2}$ for the 60 events in the study. Figure 13 presents the amplitudes and direction of the plasma drift velocities $V_{x}$ that occur in response to IP shocks (in red - sunward directions; in blue - tailward directions). The direction of the $V_{x}$ component of plasma flow is in agreement with the direction of the $E_{y} \mathrm{com}$ ponent (except for three peculiar events) and is antisunward at all local times except the nightside magnetosphere, where it is sunward near the Sun-Earth line. The tailward velocities are associated with tailward magnetic field line motion in the dayside magnetosphere. Numbers show that the magnitudes of the flow velocities $V_{x}$ range from 0.2 to $40 \mathrm{~km} \mathrm{~s}^{-1}$ and are a factor of 5 to 10 times stronger near noon as they correspond to greater variations of the electric field in this region. 
(a)

\section{(-) Pulse}

19 August 2014

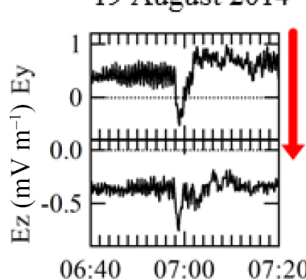

(e)

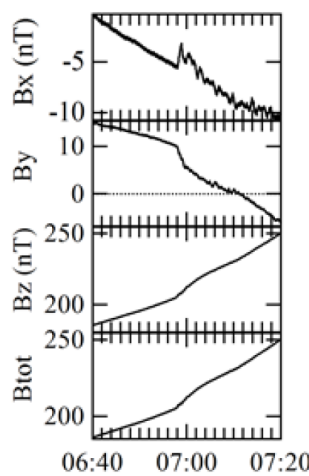

(b)
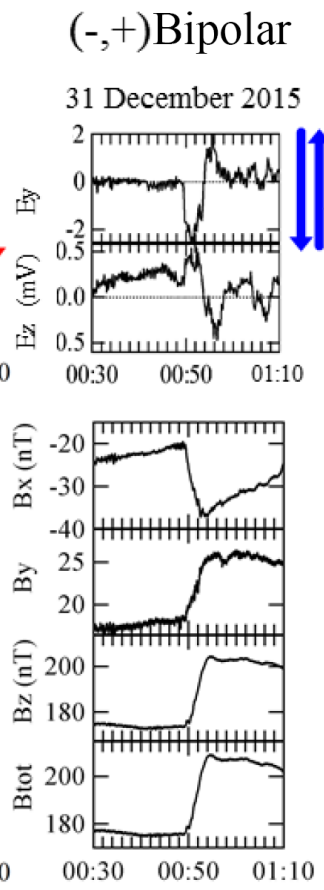

(c)

(+) Pulse

17 March 2015
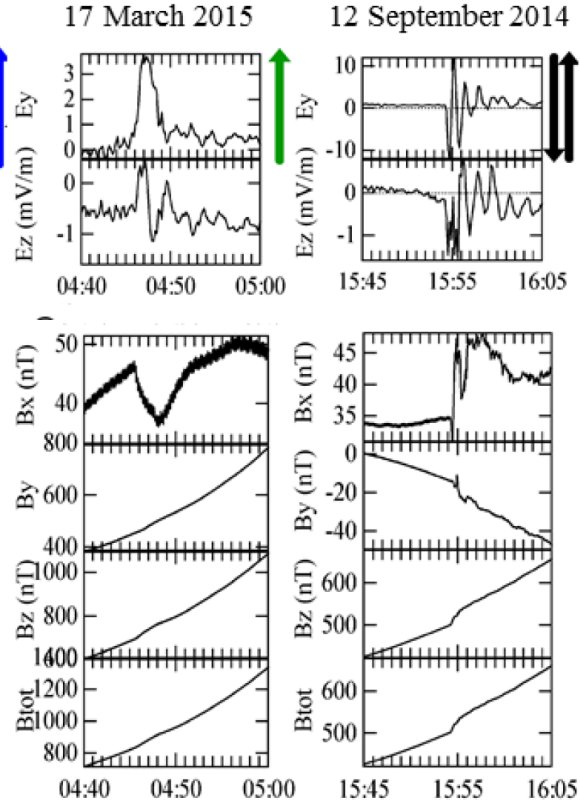

(d)

\section{Pulsations}

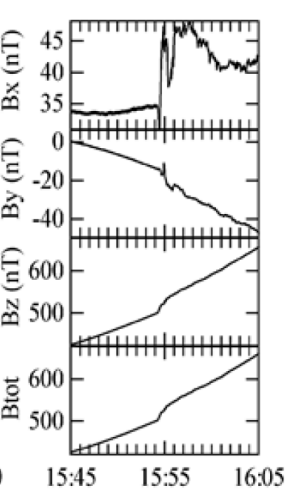

Figure 8. Examples of observed $E_{y}$ initial variation, including a negative pulse (a) a negative-positive waveform (b), a positive pulse and pulsations (a-d), and the corresponding magnetic field response (e).

(-) Pulse

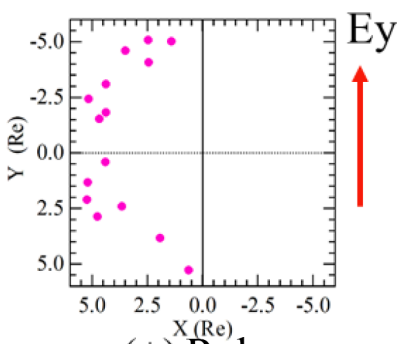

$(+)$ Pulse

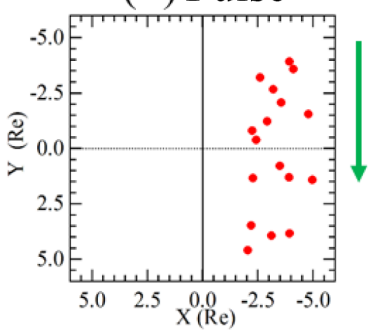

$(-,+)$ Bipolar

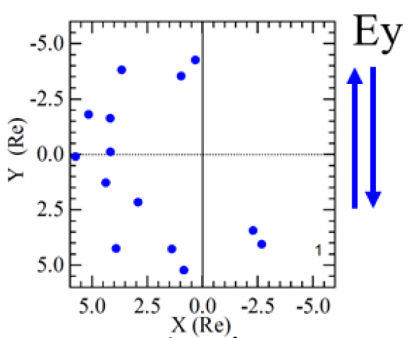

Pulsations

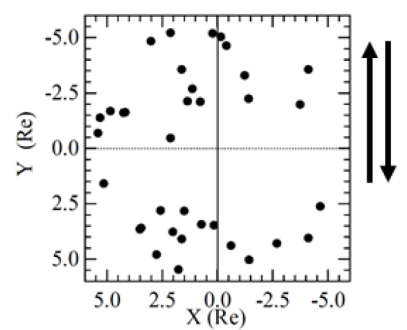

Figure 9. GSM locations where events in each of the four groups were observed in the $X-Y$ plane.

Our results are consistent with the results of global 3D MHD code simulation for the geosynchronous magnetic field response in the nightside magnetosphere to IP shocks

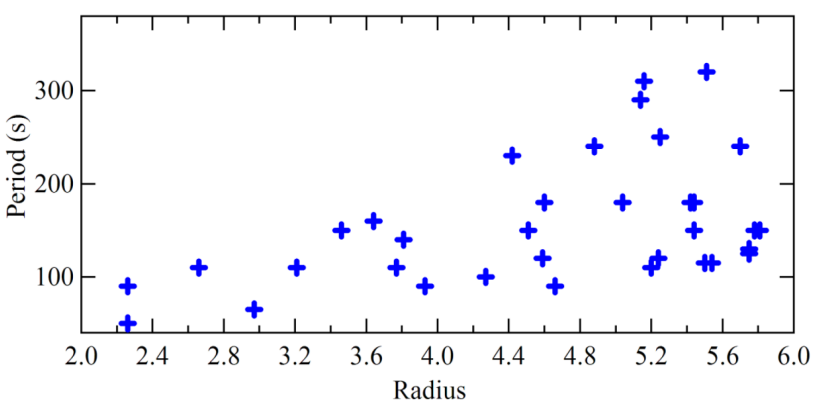

Figure 10. Periods of pulsations, initiated by IP shocks as a function of radius.

by Wang et al. (2010), presented in Fig. 14. The figure shows contours of $\delta \operatorname{abs}\left(B_{z}\right)$ and velocity vectors in the equatorial plane (blue regions $-B_{z}$ negative; red regions $-B_{z}$ positive). Their model revealed that when an IP shock sweeps over the magnetosphere there are mainly two regions in the nightside magnetosphere, a positive response region in $B_{z}$ caused by the compressive effect of the shock and a negative response region (blue), which is associated with the temporary enhancement of earthward convection. They believe that the displacement of the nightside magnetopause caused by the IP shock launches a flow in the magnetosphere near the magnetopause that has a significant $y$ component, and converges toward the $X$ axis. In the vicinity of the Sun-Earth line at 


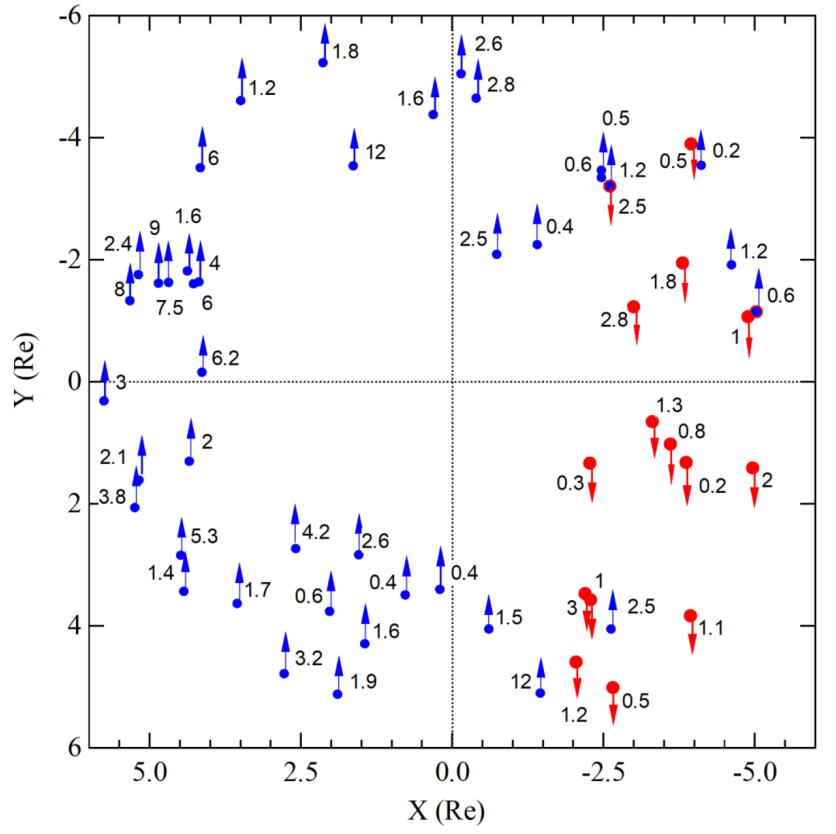

Figure 11. Amplitudes and direction of initial $E_{y}$ response to IP shocks in the $X-Y$ GSM plane (red - dawn-duskward direction; blue - duskward-dawn direction).

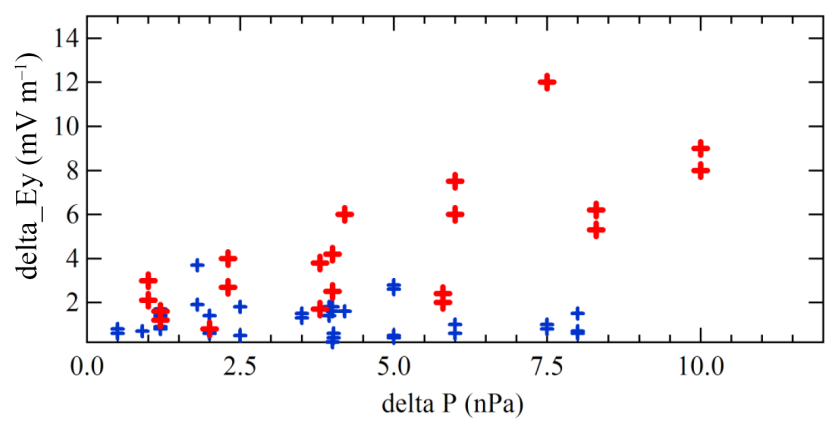

Figure 12. Amplitudes of initial $E_{y}$ variations (blue and red points) caused by a shock as a function of intensity variations of dynamic pressure observed at Wind for 60 events.

$\sim-5,-6 \operatorname{Re}$ the flow diverges, producing both an earthward flow (consistent with the sunward direction of plasma flow in the nightside magnetosphere, presented in Fig. 13) and a tailward flow.

As the direction of the shock normal should determine the direction of propagation of transient perturbations and expected flow direction in the magnetosphere initiated by an IP shock, we calculated plasma drift velocities $V_{y}$ for 30 events for which the $E_{x}$ component could be obtained from $E \cdot B=0$. We categorized them into two groups for spiral and orthospiral orientation of the shock normal. Figure 15 presents the amplitudes and direction of the plasma drift velocities $V_{y}$ observed by Van Allen Probes A and B in response to IP shocks (red - sunward $V_{x}$ directions; blue

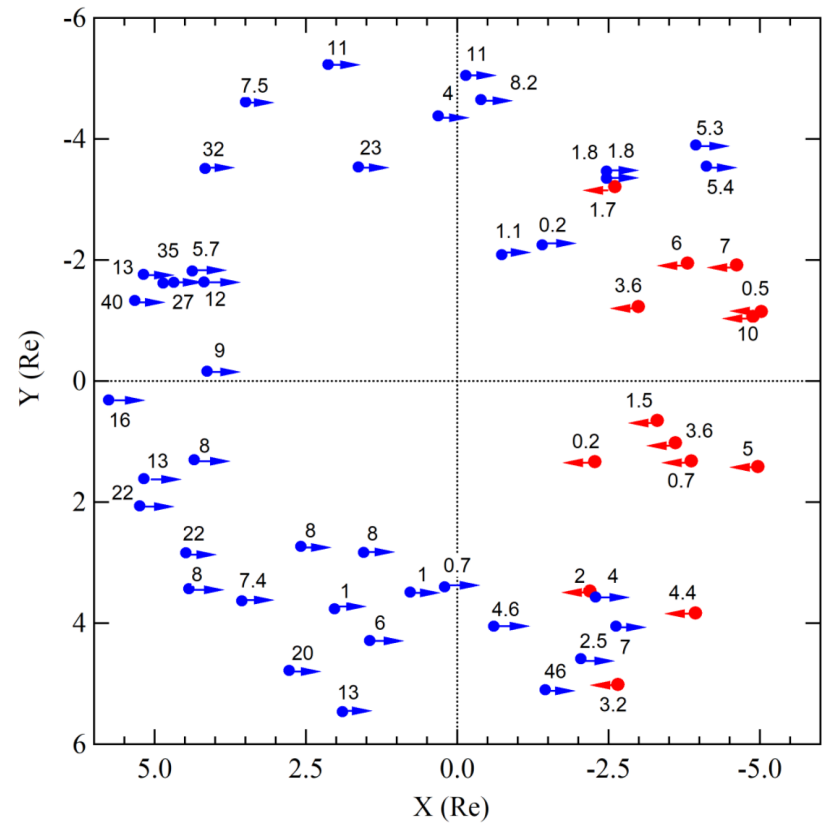

Figure 13. Amplitudes and direction of the plasma drift velocities $V_{x}=E \times B / B^{2}$ observed by Van Allen Probes A and B in response to interplanetary shocks (red - sunward direction; blue - tailward direction).

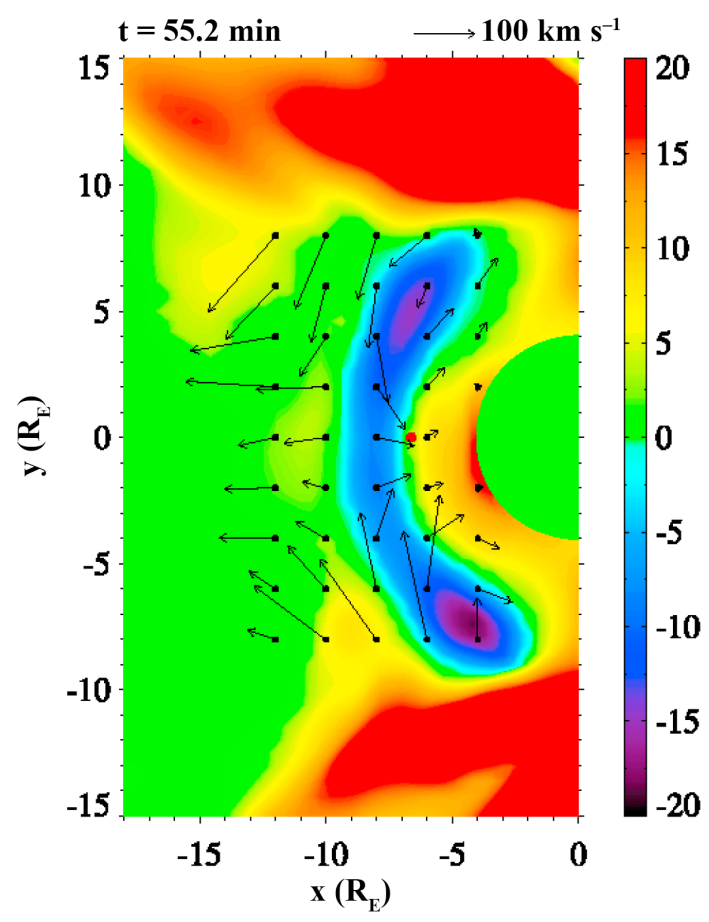

Figure 14. Results of nightside geosynchronous magnetic field response from the global MHD code simulation of IP shock (Wang et al., 2010). The arrows represent velocity vectors on the equatorial plane. 


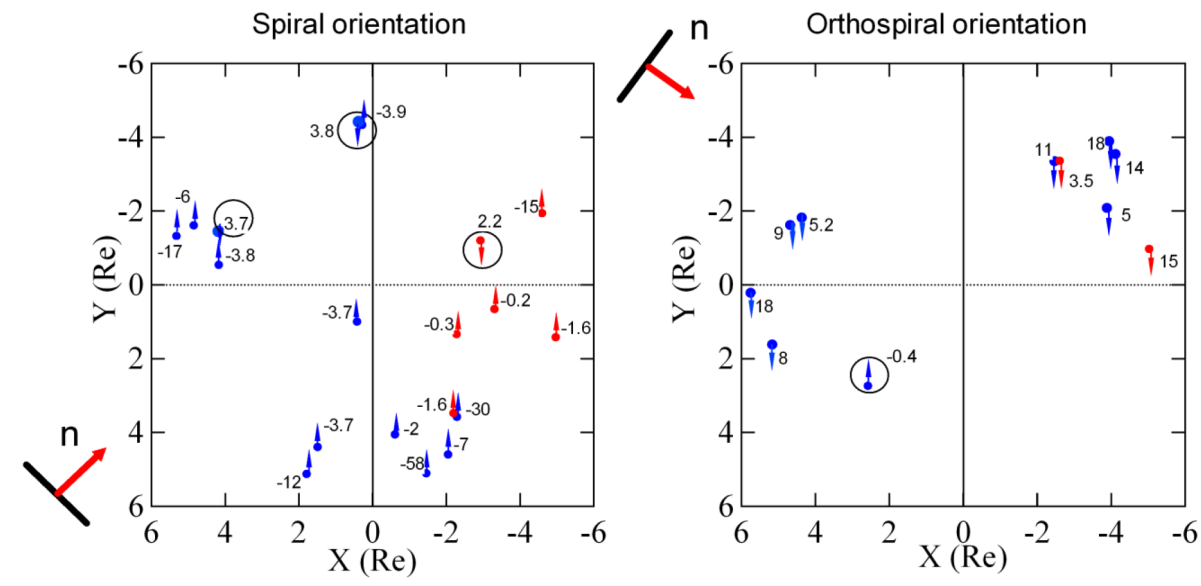

Figure 15. Amplitudes and direction of $V_{y}$ plasma drift velocities observed by Van Allen Probes A and B in response to interplanetary shocks for spiral and orthospiral orientations (red - sunward direction; blue - tailward $V_{x}$ direction).

- tailward $V_{x}$ directions) for spiral and orthospiral orientations of IP shocks. We excluded several events from the list of shocks that lacked a well defined shock normal. As anticipated, the shock orientation controls the sense of dawn or dusk flows in the magnetosphere. The observed directions of velocity $V_{y}$ predominately agree with those expected for the given shock normal orientation: dawnward for shocks that sweep dawnward across the magnetosphere, duskward for shocks that sweep duskward.

\section{Conclusions}

We presented multipoint observations concerning the response of the electric and magnetic fields, plasma and particles in the magnetosphere to an IP shock on 27 February 2014. We used a multi-spacecraft timing method to determine the propagation speed and direction of the wave front induced by the IP shock. The propagation velocity of the disturbances was about $1348 \mathrm{~km} \mathrm{~s}^{-1}$ between GOES 13 and 15 in the outer magnetosphere, but it was only about $390 \mathrm{~km} \mathrm{~s}^{-1}$ between Van Allen Probes B and A in the inner magnetosphere, consistent with expectations for a plasmasphere with limited radial extent. We deduced that the initial encounter of the IP shock with the magnetopause occurred on the postnoon magnetosphere and the shock-induced impulse propagated as a fast mode wave both dawnward and, presumably, duskward from the point of origin, consistent with the spiral orientation of the IP shock. The multipoint measurements provide evidence for a dusk-dawn oscillatory electrical field in the dayside equatorial magnetosphere with a peak-to-peak amplitude of $\sim 15 \mathrm{mV} \mathrm{m}^{-1}$ for a period of $30 \mathrm{~min}$. Both spacecraft observed enhanced fluxes of energetic electrons in the range of energies from 31.5 to $342 \mathrm{KeV}$ and their intensity shows a regular periodicity, with periods corresponding to the electric field pulsations. We interpret these observations as evidence for prompt energization of electrons due to shock-induced ULF electric fields with an additional contribution for the initial acceleration from the compressional effect of the shock. An encounter with the observed electric field for a period of $240 \mathrm{~s}$ will transport the electrons earthward by $\delta \mathrm{Re}=1.3$ to $1.6 \mathrm{Re}$ from their original positions at $L=6.4$ for Van Allen Probe A and at $L=7.1$ at Van Allen Probe B. Conservation of the first adiabatic invariant implies that such a particle will be energized by a factor of about 1.92.3 in only one cycle of the electric field pulsations. The initial plasma flow velocity in the magnetosphere was directed tailward and dawnward, consistent with expectations for the spiral orientation of the IP shock.

We identified more than 60 events observed by Van Allen Probes A and B associated with FF IP shocks for the period from 2013 to 2015. The shocks arrived from Wind with lag times in the time range from 26 to $58 \mathrm{~min}$ and produced magnetic field perturbations in the magnetosphere from several to $130 \mathrm{nT}$. We found that shocks triggered a substorm in the magnetosphere in 17 of the 30 examined events. Taking advantage of the multipoint Van Allen Probe observations, we performed a statistical study of $E_{y}$ variations of the electric field and associated plasma drift $V_{x}$ and $V_{y}$ flow velocities during the passage of interplanetary shocks. The $E_{y}$ perturbations are negative (dusk-to-dawn) in the dayside magnetosphere (followed by positive or oscillatory perturbations) and dominantly positive (dawn-to-dusk direction) in the nightside magnetosphere, particularly near the SunEarth line within an L-shell range from 2.5 to 5. The typical observed amplitudes range from 0.2 to $6 \mathrm{mV} \mathrm{m}^{-1}$ but can reach $12 \mathrm{mV}$ during strong magnetic storms. We showed that electric field perturbations increase with solar wind pressure and that the changes are especially marked in the dayside magnetosphere. The direction of the $V_{x}$ component of plasma flow is in agreement with the direction of the $E_{y}$ component and is antisunward at all local times except the nightside magnetosphere, where it is sunward near the Sun- 
Earth line but antisunward towards dawn and dusk. The flow velocities $V_{x}$ range from 0.2 to $40 \mathrm{~km} \mathrm{~s}^{-1}$ and are a factor of 5 to 10 times stronger near noon as they correspond to greater variations of the electric field in this region. We investigated how the electric field perturbations deviate from the preceding undisturbed period and demonstrated that the shock-induced electric field signatures can be classified into four different groups according to the initial $E_{y}$ electric field response. These signatures are dependent on local time. Negative and bipolar pulses predominate on the dayside while positive pulses occur on the nightside. The ULF electric field pulsations of Pc and Pi types produced by IP shocks are observed at all local times and in the range of periods from several tens of seconds to several minutes. We believe that most electric field pulsations of the Pc5 type in the dayside magnetosphere at $L<6$ are produced by field line resonances. One of the most important results from the present study is that the direction of the shock normal determines the direction of the propagation of the shock-induced magnetic and plasma disturbances. The observed directions of velocity $V_{y}$ predominately agree with those expected for the given spiral or orthospiral shock normal orientation. Our results are consistent with the results of global MHD code simulation of the geosynchronous nightside magnetic field response to IP shock by Wang et al. (2010).

Data availability. Data used in the paper are available publicly at http://cdaweb.gsfc.nasa.gov/istp_public/ (Coordinated Data Analysis Web, NASA, 2018). GOES data can be found at http:// satdat.ngdc.noaa.gov/sem/goes/data/new_full/ (NOAA, 2018). The electric field data were obtained from http://www.space.umn.edu/ rbspefw-data (Wygant and Breneman, 2017). The list of IP shocks used in this study was obtained from site http://ipshocks.fi (University of Helsinki, 2018).

Author contributions. GK drafted and wrote the paper and all others commented it. GK, DS, ST and RR were the primary contributors to this work. DS initiated the idea and collaboration. ST programming, software development, consulting regarding use of Van Allen electric field data. RR - programming, software development, consulting regarding use of GOES magnetic field data. JW - consulting regarding use of Van Allen electric field data. HS consulting regarding use of Van Allen plasma data. CK - consulting regarding use of Van Allen Magnetometer data. VA - consulting regarding use of THEMIS data.

Competing interests. The authors declare that they have no conflict of interest.

Acknowledgements. The Van Allen Probes mission is supported by NASA. NASA GSFC's CDAWEB provided Wind and observations, while SSCWEB provided Van Allen Probes EPHEMERIS. The work by GIK at the University of Maryland was supported by grants from NASA NNX15AW86G S01 and NSF AGS-1207445. The work by the EFW team at the University of Minnesota was supported by APL contract to UMN 922613 under NASA contract to APL NAS5-01072.

Edited by: Vincent Maget

Reviewed by: three anonymous referees

\section{References}

Abraham-Shrauner, B. and Yun, S. H.: Interplanetary shocks seen by Ames Plasma Probe on Pioneer 6 and 7, J. Geophys. Res., 81, 2097-2102, https://doi.org/10.1029/JA081i013p02097, 1976.

Araki, T.: A physical model of the geomagnetic sudden commencement, AGU Geophys. Monogr., 81, 183-200, 1994.

Auster, H. U., Glassmeier, K.-H., Magnes, W., Aydogar, O., W. Baumjohann, W., Constantinescu, D., Fischer, D., Fornacon, K. H., Georgescu, E., Harvey, P., Hillenmaier, O., Kroth, R., Ludlam, M., Narita, Y., Nakamura, R., Okrafka, K., Plaschke, F., Richter, I., Schwarzl, H., Stoll, B., Valavanoglou, A., and Wiedemann, M.: The THEMIS Fluxgate Magnetometer, Space Sci. Rev., 141, 235-264, https://doi.org/10.1007/s11214-008-9365-9, 2008.

Balogh, A., Dunlop, M. W., Cowley, S. W. H., Southwood, D. J., Thomlinson, J. G., Glassmeier, K. H., Musmann, G., Lohr,H., Buchert, S., Acuña, M. H., Fairfield, D. H., Slavin, J. A., Riedler, W., K. Schwingenschuh, M.G. Kivelson, M. G., and the Cluster Magnetometer Team: The Cluster Magnetic Field Investigation, Space Sci. Rev., 79, 65-92, 1997.

Blake, J. B., Carranza, P. A., Claudepierre, S. G., Clemmons, J. H., Crain Jr., W. R., Dotan, Y., Fennell, J. F., Fuentes, F. H., Galvan, R. M., George, J. S., Henderson, M. G., Lalic, M., Lin, A. Y., Looper, M. D., Mabry, D. J., Mazur, J. E., McCarthy, B., Nguyen, C. Q., O’Brien, T. P., Perez, M. A., Redding, M. T., Roeder, J. L., Salvaggio, D. J., Sorensen, G. A., Spence, H. E., Yi, S., and Zakrzewski, M. P.: The Magnetic Electron Ion Spectrometer (MagEIS) Instruments Aboard the Radiation Belt Storm Probes (RBSP) Spacecraft, Space Sci. Rev., 179, 383-421, https://doi.org/10.1007/s11214-013-9991-8, 2013.

Blake, J. B., Kolasinski , W. A., Fillius, R. W., and Mullen, E. G.: Injection of electrons and protons with energies of tens of $\mathrm{MeV}$ into $L<3$ on 24 March 1991, Geophys. Res. Lett., 19, 821-824, https://doi.org/10.1029/92GL00624, 1992

Claudepierre, S. G., Mann, I. R., Takahashi, K., Fennell, J. F., Hudson, M. K., Blake, J. B., Roeder, J. L., Clemmons, J. H., Spence, H. E., Reeves, G. D., Baker, D. N., Funsten, H.O., Friedel, R. H. W., Henderson, M. G., Kletzing, C. A., Kurth, W. S., MacDowall, R. J., Smith, C. W., and Wygant, J. R.: Van Allen Probes Observation of Localized Drift-Resonance Between Poloidal Mode Ultra-low Frequency Waves and $60 \mathrm{keV}$ Electrons, Geophys Res. Lett., 40, 4491-4497, https://doi.org/10.1002/grl.50901, 2013.

Colburn, D. C. and Sonett, C. P.: Discontinuities in the solar wind, Space Sci. Rev., 5, 439-506, 1966.

Foster, J., Wygant, J., Hudson, M., Boyd, A., Baker, D., Erickson, P., and Spence, H. E.: Shock-induced prompt relativistic electron acceleration in the inner magnetosphere, J. Geophys. Res. 120, 1661-1674, https://doi.org/10.1002/2014JA020642, 2015. 
Glassmeier, K. H. and Heppner, C.: Travelling magnetospheric twin-vortices: Another case study, global characteristics and a model, J. Geophys. Res., 97, 3977-3992, https://doi.org/10.1029/91JA02464, 1992.

Halford, A. J., McGregor, S. L., Murphy, K. R., Millan, R. M., Hudson, M. K., Woodger, C. A., Cattel, L. A., Breneman, A. W., Mann, I. R., Kurth, W. S., Hospodarsky, G. B., Gkioulidou, M., and Fennell, J. F.: BARREL observations of an ICME-Shock impact with the magnetosphere and the resultant radiation belt electron loss, J. Geophys. Res.-Space, J. Geophys. Res., 120, 25572570, https://doi.org/10.1002/2014JA020873, 2015.

Hao, Y., Zong, Q.-G., Wang, Y., Zhou, X.-Z., Zhang, H., Fu, S., $\mathrm{Pu}, \mathrm{Z}$. Y., Spence, H. E., Blake, J. B., Bonnell, J., Wygant, J., and Kletzing, C.: Interactions of energetic electrons with ULF waves triggered by interplanetary shock: Van Allen Probes observations in the magnetotail, J. Geophys. Res., 119, 8262-8273, https://doi.org/10.1002/2014JA020023, 2014.

Kanekal, S. G., Baker, D. N., Fennell, J. F., Jones, A., Schiller, Q., Richardson, I. G., Li, X., Turner, D. L., Califf, S., Claudepierre, S. G., Wilson III, L. B., Jaynes, A.,. Blake, J. B., Reeves, G., Spence, H. E., Kletzing, C. A., and Wygant, J. R.: Prompt Acceleration of Magnetospheric Electrons to Ultra-Relativistic Energies by the 17 March 2015 Interplanetary Shock, J. Geophys. Res., 121, 7622-7635, https://doi.org/10.1002/2016JA022596, 2016.

Kim, K.-H., Park, K. S., Ogino, T., Lee, D.-H., Sung, S.-K., and Kwak Y.-S.: Global MHD simulation of the geomagnetic sudden commencement on 21 October 1999, J. Geophys. Res., 114, A08212, https://doi.org/10.1029/2009JA014109, 2009.

Kletzing, C. A., Kurth, W. S., Acuna, M., MacDowall, R. J, Torbert, R. B., Averkamp, T., Bodet, D., Bounds, S. R., Chutter, M., Connerney, J., Crawford, D., Dolan, J. S., Dvorsky R., Hospodarsky, G. B., Howard, J., Jordanova, V., Johnson, R. A., Kirchner, D. L., Mokrzycki, B., Needell, G., Odom, J., Mark, D., Pfaff Jr., Phillips, J. R. , Piker, C.W., Remington, S. L., Rowland, D., Santolik, O., Schnurr, R., Sheppard, D., Smith, C. W., Thorne, R. M., and Tyler, J. J.: The Electric and Magnetic Field Instrument Suite and Integrated Science (EMFISIS) on RBSP, Space Sci. Rev., 179, 127-181, https://doi.org/10.1007/s11214-013-9993-6, 2013.

Knott, K., Fairfield, D., Korth, A., and Young, D. T.: Observations near the magnetopause at the onset of the July 29, 1977, sudden storm commencement, J. Geophys. Res., 87, 5888-5894, https://doi.org/10.1029/JA087iA08p05888, 1982.

Lepping, R. P., Acuna, M. H., Burlaga, L. F., Farrell, W. M., Slavin, J. A.,Schatfen, K. H., Mariani, E., Ness, N. E., Neubauer, E. M., Whang, Y. C., Byrnes J. B., Kennon R. S., Panetta, P. V., Scheifele J., and Worley, E. M.: The WIND Magnetic field investigation, Space Sci Rev., 71, 207-229, 1995.

Li, X., Roth, I., Temerin, M., Wygant, J. R., Hudson, M. K., and Blake, J. B.: Simulation of the prompt energization and transport of radiation belt particles during the March 24, 1991, Geophys. Res. Lett., 20, 2423-2426, https://doi.org/10.1029/93GL02701, 1993.

Liou, K., Newell, P. T., Meng, C.-I. , Wu, C.-C., and Lepping, R. P.: Investigation of external triggering of substorms with Polar ultraviolet imager observations, J. Geophys. Res., 108, 1364-1378, https://doi.org/10.1029/2003JA009984, 2003.
Mauk, B. H., Fox, N. J., Kanekal, S. G., Kessel, R. L., Sibeck, D. G., and Ukhorskiy, A.: Science objectives and rationale for the radiation belt storm probes mission, Space Sci. Rev., 179, 3-27, https://doi.org/10.1007/s11214-012-9908-y, 2012.

McFadden, J. P., Carlson, C. W., Larson, D., Ludlam, M., Abiad, R., Elliott, B., Turin, P., Marckwordt, M., and Angelopoulos, V.: The THEMIS ESA plasma instrument and in-flight calibration, Space Sci. Rev., 141, 277-302, https://doi.org/10.1007/s11214008-9440-2, 2008.

NASA: Coordinated Data Analysis Web, available at: http:// cdaweb.gsfc.nasa.gov/istp_public/, last access: 1 August 2018

NOAA: GOES SEM fata, available at: http://satdat.ngdc.noaa.gov/ sem/goes/data/new_full/, last access: 2 January 2018.

Nopper Jr., R. W., Hughes, W. J., MacLennan, C. G., and McPherron, R. L.: Impulse-excited pulsations during the July 29, 1977, event, J. Geophys. Res., 87, 5911-5916, https://doi.org/10.1029/JA087iA08p05911, 1982.

Ogilvie, K. W., Chornay, D. J., Fritzenreiter, R. J., Hunsaker, F., Keller, J., Lobell, J., Miller, G., Scudder, J. D., Sittler Jr., E.C., Torbert, R. B., Bodet, D., Needell, G., Lazarus, A. J., Steinberg, J. T., Tappan, J. H., Mavretic, A., and Gergin, E.: A comprehensive plasma instrument for the WIND spacecraft, Space Sci. Rev., 71, 55-77, https://doi.org/10.1007/BF00751326, 1995.

Oliveira, D. M. and Raeder, J.: Impact angle control of interplanetary shock geoeffectiveness: A statistical study, J. Geophys. Res., 120, 4313-4323, https://doi.org/10.1002/2015JA021147, 2015.

Samsonov, A. A., Sibeck, D. G., and Imber, J.: MHD simulation for the interaction of an interplanetary shock with the Earth's magnetosphere, J. Geophys. Res., 112, A12220, https://doi.org/10.1029/2007JA012627, 2007.

Schiller, O., Kanekal, S. G., Jian, L. K., Li, X., Jones, A., Baker, D. N., Jaynes, A., and Spence, H. E.: Prompt injections of highly relativistic electrons induced by interplanetary shocks: A statistical study of Van Allen Probes observations, Geophys. Res. Lett., 43, 12317-12324, https://doi.org/10.1002/2016GL071628, 2016.

Schmidt, R. and Pedersen, A.: Signatures of storm sudden commencements in the electric field measured at geostationary orbit (GEOS-2), Phys. Scr., 37, 491-495, 1988.

Shinbori, A., Ono, T., Iizima, M., Kumamoto, A., and Oya, H.: Sudden commencements related plasma waves observed by the Akebono satellite in the polar region and inside the plasmasphere region, J. Geophys. Res., 108, 1457, https://doi.org/10.1029/2003JA009964, 2003.

Shinbori, A., Ono, T., Iizima, M., and Kumamoto, A.: SC related electric and magnetic field phenomena observed by the Akebono satellite inside the plasmasphere, Earth Planet. Space, 56, 269282, 2004.

Sibeck, D. G.: A model for the transient magnetospheric response to sudden solar wind dynamic pressure variations, J. Geophys. Res., 95, 3755-3771, https://doi.org/10.1029/JA095iA04p03755, 1990.

Singer, H. J., Matheson, L., Grubb, R., Newman, A., and Bouwer, S. D.: Monitoring space weather with the GOES magnetometers, Proc. SPIE Int. Soc. Opt. Eng., 2812, 299-308, 1996.

Southwood, D. J. and Kivelson, M. G.: Charged particle behavior in low-frequency geomagnetic pulsations: 1. Transverse waves, J. Geophys. Res., 86, 5643-5655, https://doi.org/10.1029/JA086iA07p05643, 1981. 
Southwood, D. J. and Kivelson, M. G.: The Magnetohydrodynamic Response of the Magnetospheric Cavity to Changes in Solar Wind Pressure, J. Geophys. Res., 95, 2301-2309, https://doi.org/10.1029/JA095iA03p02301, 1990.

Spence, H. E., Reeves, G. D., Baker, D. N., Blake, J. B., Bolton, M., Bourdarie, S., Chan, A. H. S., Claudepierre, G., Clemmons, J. H., Cravens, J. P., Elkington, S. R., Fennell, J. F., Friedel, R. H. W., Funsten, H. O., Goldstein, J., Green, J. C., Guthrie, A., Henderson, M. G , Horne, R. B., Hudson, M. K., Jahn, J.M., Jordanova, V. K., Kanekal, S. G., Klatt, B. W., Larsen, B. A., Li, X., MacDonald, E. A., Mann, I. R., Niehof, J., O’Brien, T. P., Onsager, T. G., Salvaggio, D., Skoug, R. M., Smith, S. S., Suther, L. L., Thomsen, M. F., and Thorne, R. M.: Science Goals and Overview of the Energetic Particle, Composition, and Thermal Plasma (ECT) Suite on NASA's Radiation Belt Storm Probes (RBSP) Mission, Space Sci. Rev., 179, 311-336, https://doi.org/10.1007/s11214-013-0007-5 2013.

Takahashi, N., Kasaba, Y., Nishimura, Y., Shinbori, A., Kikuchi, T., Hori, T., Ebihara, Y., and Nishitani, N.: Propagation and evolution of electric fields associated with solar wind pressure pulses based on spacecraft and ground-based observations, J. Geophys. Res.-Space Phys., 122, 8446-8461, https://doi.org/10.1002/2017JA023990, 2017.

Tamao, T.: The structure of three-dimensional hydromagnetic waves in a uniform cold plasma, J. Geomagn. Geoelectr., 48, 89114, https://doi.org/10.5636/jgg.16.89, 1964.

University of Helsinki: Database of Heliospheric Shock Waves, available at: http://ipshocks.fi, last access: 1 September 2018.

Wang, C., Sun, T. R., Guo, X. C., and Richardson, J. D.: Case study of nightside magnetospheric magnetic field response to interplanetary shocks, J. Geophys. Res., 115, A10247, https://doi.org/10.1029/2010JA015451, 2010.

Wilken, W., Goertz, C. K., Baker, D. N., Higbie, P. R., and Fritz , T. A.: The SSC on July 29, 1977 and its propagation within the magnetosphere, J. Geophys. Res., 87, 5901-5910, https://doi.org/10.1029/JA087iA08p05901, 1982.
Wilken, W., Baker, D. N., Higbie, P. R., Fritz, T. A., Olson, W. P., and Pfitzer, K. A.: Magnetospheric configuration and energetic particle effects associated with a SSC: A case study of the CDAW event on March 22, 1979, J. Geophys. Res., 91, 14591473, https://doi.org/10.1029/JA091iA02p01459, 1986.

Wygant, J. and Brenemean, A.: RBSP/EFW Home - University of Minnesota, available at: http://www.space.umn.edu/ rbspefw-data, last access: 1 September 2017.

Wygant, J., Mozer, F., Temerin, M., Blake, J., Maynard, N., Singer, H., and Smiddy M.: Large amplitude electric field and magnetic field signatures in the inner magnetosphere during injection of $15 \mathrm{MeV}$ electron drift echoes, Geophys. Res. Lett., 21, 17391742, https://doi.org/10.1029/94GL00375, 1994.

Wygant, J. R., Bonnell, J. W., Goetz, K., Ergun, R. E., Mozer, F. S., Bale, S. D., Ludlam, M., Turin, P., Harvey, P. R., Hochmann, R., Harps, K., Dalton, G., McCauley, J., Rachelson, W., Gordon, D., Donakowski, B., Shultz, C., Smith, C., Diaz-Aguado, M., Fischer, J., Heavner, S., Berg, P., Malaspina, D. M., Bolton, M. K., Hudson, M., Strangeway, R. J., Baker, D. N., Li, X., Albert, J., Foster, J. C., Chaston, C. C., Mann, I., Donovan, E., Cully, C. M., Cattell, C. A., Krasnoselskikh, V., Kersten, K., Breneman, A., and Tao, J. B.: The electric field and waves instruments on the radiation belt storm probes mission, Space Sci. Rev., 179, 183-220, https://doi.org/10.1007/s11214-013-0013-7, 2013.

Zong, Q.-G., Zhou, X.-Z., Wang, Y. F., Li, X., Song, P., Baker, D. N., Fritz, T. A., Daly, P. W., Dunlop, M., and Pedersen, A.: Energetic electron response to ULF waved induced by interplanetary shocks in the outer radiation belt, J. Geophys. Res., 114, A10204, https://doi.org/10.1029/2009JA014393, 2009. 